\title{
Mapping the landscape of tandem repeat variability by targeted long read single molecule sequencing in familial X-linked intellectual disability
}

\author{
Alena Zablotskaya ${ }^{1}$, Hilde Van Esch ${ }^{2}$, Kevin J. Verstrepen ${ }^{3}$, Guy Froyen ${ }^{4}$ and Joris R. Vermeesch ${ }^{{ }^{*}}$ (D)
}

\begin{abstract}
Background: The etiology of more than half of all patients with X-linked intellectual disability remains elusive, despite array-based comparative genomic hybridization, whole exome or genome sequencing. Since short read massive parallel sequencing approaches do not allow the detection of larger tandem repeat expansions, we hypothesized that such expansions could be a hidden cause of X-linked intellectual disability.

Methods: We selectively captured over 1800 tandem repeats on the X chromosome and characterized them by long read single molecule sequencing in 3 families with idiopathic $X$-linked intellectual disability.

Results: In male DNA samples, full tandem repeat length sequences were obtained for 88-93\% of the targets and up to $99.6 \%$ of the repeats with a moderate guanine-cytosine content. Read length and analysis pipeline allow to detect cases of $>900 \mathrm{bp}$ tandem repeat expansion. In one family, one repeat expansion co-occurs with downregulation of the neighboring MIR222 gene. This gene has previously been implicated in intellectual disability and is apparently linked to FMR1 and NEFH overexpression associated with neurological disorders.

Conclusions: This study demonstrates the power of single molecule sequencing to measure tandem repeat lengths and detect expansions, and suggests that tandem repeat mutations may be a hidden cause of X-linked intellectual disability.
\end{abstract}

Keywords: Tandem repeats, Expansion, Single molecule sequencing, X-linked intellectual disability

\section{Background}

Intellectual disability (ID) has a prevalence of $2.3 \%$, making it a prime socio-economical problem [1]. ID is a very complex and heterogeneous disorder that can be caused by genetic factors, environmental factors or a combination of both. As a result, the etiology remains unknown in $\sim 30 \%$ of cases. X chromosome-linked ID (XLID) has served as a model for the genetics underlying ID, in part because it is approximately $30 \%$ more prevalent in males than in females, suggesting that important causative genetic loci are located on the X-chromosome [2].

\footnotetext{
* Correspondence: joris.vermeesch@kuleuven.be

${ }^{1}$ Department of Human Genetics and Center for Human Genetics, Laboratory for Cytogenetics and Genome Research, University Hospitals Leuven, KU Leuven, O\&N I Herestraat 49 - box 606, 3000 Leuven, Belgium Full list of author information is available at the end of the article
}

In the last 15 years, candidate gene mutation screening $[3,4]$, hybridization-based array screens $[5,6]$ including high resolution array-CGH [7-10], and massively-parallel sequencing (MPS) screens [11-14] led to the identification of many genes associated with ID. It became clear that genetic causes of ID are highly heterogeneous, as the reported mutations explain only a small number of ID families [2]. For example, a Sanger sequencing-based screen of all exons on the X-chromosome in 208 XLID families only revealed causal mutations in $25 \%$ of families [15]. Later, MPS allowed for much higher throughput identification of disease-associated mutations, deletions and duplications. However, this groundbreaking method could not resolve more than $20 \%$ of the remaining cases, as illustrated by an X chromosome-specific exome MPS screen in 405 XLID families [14]. Thus, despite the large number

(c) The Author(s). 2018 Open Access This article is distributed under the terms of the Creative Commons Attribution 4.0 International License (http://creativecommons.org/licenses/by/4.0/), which permits unrestricted use, distribution, and reproduction in any medium, provided you give appropriate credit to the original author(s) and the source, provide a link to the Creative Commons license, and indicate if changes were made. The Creative Commons Public Domain Dedication waiver (http://creativecommons.org/publicdomain/zero/1.0/) applies to the data made available in this article, unless otherwise stated. 
of studies and significant technological progress, the etiology of ID remains unsolved in at least $40 \%$ of XLID families.

These figures strongly suggest that the missing mutations should be searched for in the non-coding regions of the $\mathrm{X}$ chromosome, or in regions that currently escape analysis. An intergenic variant identified by targeted MPS on the complete linkage interval of a large XLID family has been associated with enhanced expression of $H C F C 1$ in a family with nonsyndromic ID [16] demonstrating that indeed regulatory mutations contribute to ID.

Another group of regions that have been neglected are the tandem repeats. Tandem repeats largely escape mutation analysis because their larger sizes are not covered by short read sequencing technologies. In addition, the sequence reads often fail to be mapped back to the reference genome due to their repetitive nature.

Tandem repeats are DNA sequences consisting of multiple (almost) identical copies of a short (typically 1-50 nt) unit sequence that is repeated in a head-to-tail manner. Such repeats are arbitrarily divided into microsatellites and minisatellites, depending on repeat unit length. Tandem repeats are abundantly present in the human genome including the coding sequences and promoters [17], but thorough variation analysis is lacking due to technical challenges. The mutation rate of repeat regions is typically at least one order of magnitude higher than those in non-repetitive DNA, and as a consequence, variation in repeat length in coding or regulatory regions has a high probability to influence the function or expression of genes [18-20]. Despite all those features, repeats are often overlooked as prime targets for disease-related mutations. Moreover, the most commonly used MPS instruments from Illumina and Thermofisher provide average read lengths of $150-200 \mathrm{nt}$, which is too short to read through most repeats. Even paired-end sequencing does not increase read length in this case, because in order to obtain reliable sequences both reads of a pair should span a full tandem repeat with flanks. Therefore, long-read sequencing technologies are more suitable to study repeat variations. Recently, single molecule real-time sequencing has been introduced to study tandem repeats through long-range PCR amplicons spanning a single repeat of interest. Despite the significant error rate of this newest MPS technology, an accurate consensus tandem repeat can be reconstructed via a local de novo assembly [21-23]. In addition, this long read MPS platform is especially valuable to study expansions because of the circular nature of the reads. Multiple passes through the read sequence allow to generate a consensus sequence which facilitates discrimination between sequencing errors and PCR artefacts ("stutters"), that are commonly obscuring tandem repeat analyses.
To test the hypothesis that tandem repeat expansions are a hidden cause of XLID, we set out to selectively target repetitive sequences on the $\mathrm{X}$ chromosome and characterize them by single molecule sequencing using the PacBio platform. Specifically, we targeted more than 1800 tandem repeats on the X-chromosome in 3 families with idiopathic X-linked intellectual disability in whom previous methods did not detect any potential genetic cause. Our analysis identified one candidate causal repeat expansion in one family. Gene expression analysis showed down-regulation of the neighboring MIR222 and, indirectly, FMR1 and NEFH overexpression. This study suggests that tandem repeat mutations may be a hidden cause of XLID and potentially of other diseases as well.

\section{Methods}

Selection of tandem repeats and capture probe design

A list of tandem repeats on the $\mathrm{X}$ chromosome was obtained from the hg19 human reference genome (UCSC [24]) with the ETANDEM tool (EMBOSS package [25]) and was complemented by repeats from several other sources [19, 26-29] as described by Duitama et al. [30] bringing the total number of target repeats to 43,106 . They included repetitive loci with a unit size $1-50 \mathrm{bp}$, copy numbers of 2-809, full length $22-4048 \mathrm{bp}$, and GC content including the extreme values of 0 and $100 \%$.

All tandem repeats were annotated according to their position relative to a gene and divided into two groups: presumably functional (i.e. located in coding and regulatory regions) and likely non-functional as previously described [30]. The variability potential of these repeats was predicted by the SERV score [17] based on the following characteristics: unit length, copy number in the reference genome, and intralocus homology. SERV values 1-3 correspond to the highly variable tandem repeats that are usually used for genotyping.

We aimed to sequence around 2000 repeat loci on the $\mathrm{X}$ chromosome. We reasoned that a total of at least 500 flanking base pairs should be kept in a $1 \mathrm{~kb}$ consensus sequence for probe annealing sites and repeat variation, hence the maximal length threshold of $500 \mathrm{bp}$ for tandem repeats. We also kept a number of intronic and intergenic tandem repeats on the $\mathrm{X}$ chromosome to narrow down the linkage intervals with inheritance patterns of more than 2 haplotype specific alleles per repetitive locus.

First, tandem repeats were pre-selected based on their characteristics, presumed functionality and/ or predicted degree of polymorphism (Table 1): 1) 353 tandem repeats in coding regions with the total size up to $500 \mathrm{bp}$; 2) 174 tandem repeats with SERV score $\geq 1$ and total length $\leq 500 \mathrm{bp}$, which are located in regulatory sites (CpG islands, transcription factor binding sites, regions 


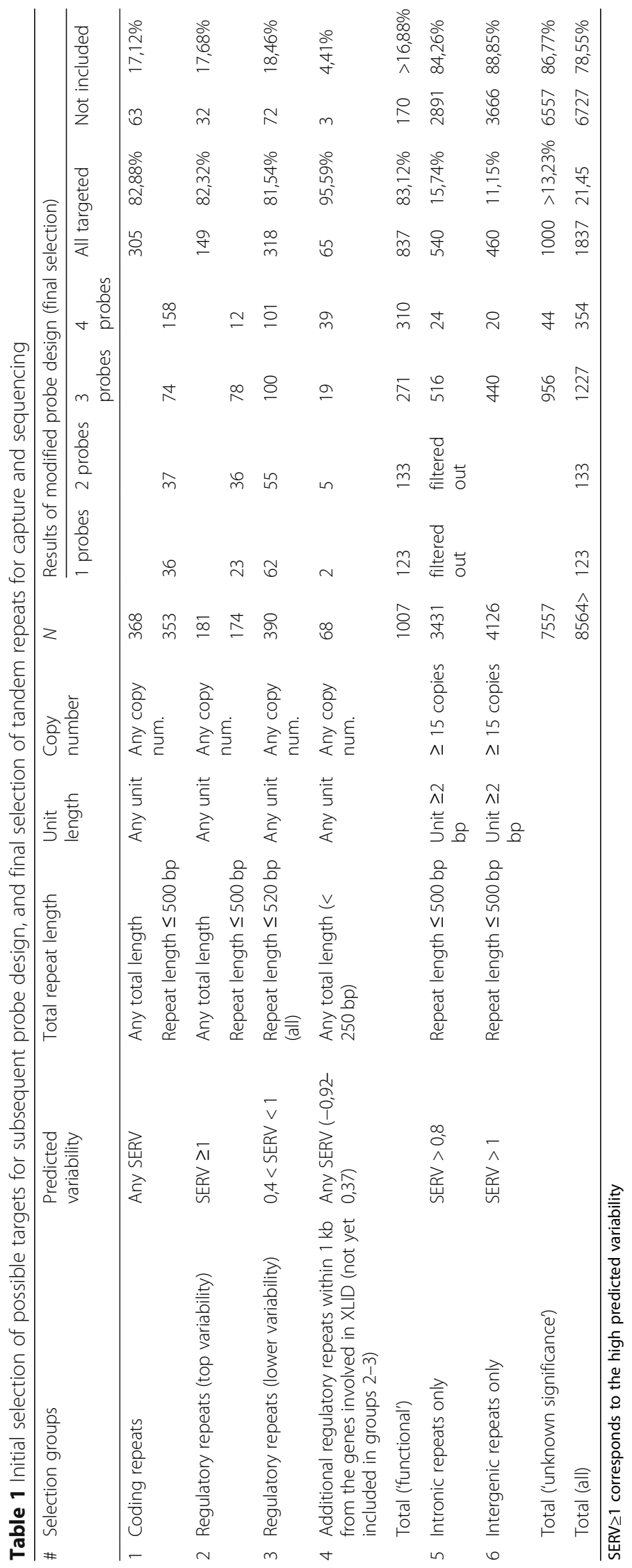


upstream or downstream from a gene, including microRNA genes); 3) 390 regulatory repeats of any size with SERV scores $0.4-1$; 4) 68 regulatory tandem repeats within $1000 \mathrm{bp}$ distance from the 112 genes that are known to be involved in XLID (Greenwood Genetic Center, [31]) and not yet included for probe design; 5) 1000 non-functional tandem repeats evenly distributed over the X chromosome with SERV score $>0.8$, at least 15 copies in the reference genome, unit length $>1 \mathrm{bp}$, total length $\leq 500 \mathrm{bp}, \mathrm{GC}$ content $30-70 \%$, and at least 3 different capture probes available.

Then probe design was performed as previously described [30] and included left and right flanking probes, spanning probes centered on tandem repeats, and double probes containing both flanks of a target. In total, 9969 probes generated for 4503 tandem repeats (715 functional and 3788 non-functional repeats) matched uniqueness criterion, i.e. were not predicted to hybridize aspecifically (Table S1). Subsequently, for those 270 repeats where it was not possible to generate unique capture probes, another round of probe design was performed with modified settings: flanking probes and two parts of a double probe were allowed to shift outwards from a repeat by up to $500 \mathrm{bp}$, and the most proximal available unique probe was chosen for the application (see Additional file 1). This approach allowed us to generate 118 new probes and add 66 tandem repeats to our final repeat selection. To increase the total number of available probes from 1 to 3 or 4 , the same strategy was applied to the 7 'XLID repeats' and was successful for 6 of them.

Finally, we examined the distribution of 204 functional repeats which could not be targeted after the above described steps. These repeats were tested for homology to other genomic loci using the Bowtie alignment tool [32] with the following settings: -e 200 -n $3-\mathrm{y}-115-\mathrm{k} 10$ (or -k 30 , depending on the expected number of locus-specific alignments). When a repeat together with its capture probes revealed a local specificity, but no homology to unrelated regions (e.g. showed homology only with other sequences within a cluster of a locally duplicated region), this repeat was kept in the final selection.

Following this approach, two distinguishable clusters of untargeted repeats were found at both ends of the $\mathrm{X}$ chromosome. Eighteen tandem repeats on the $\mathrm{p}$ arm fall in the pseudoautosomal region 1 (PAR1) with a $100 \%$ homology with the corresponding region on the $\mathrm{Y}$ chromosome. For 14 out of 18 repeats in PAR1 there was no high homology detected other than with the $\mathrm{Y}$ chromosome, and 1 to 3 locus-specific probes were available per repeat. Similarly, we reanalyzed probes for 13 repeats in PAR2 on the $\mathrm{q}$ arm, but no locus-specific probes were found due to a high similarity of these sequences to other regions on the chromosomes $\mathrm{X}, \mathrm{Y}$ or autosomes.
Two other clusters of so far untargeted tandem repeats were detected on the $\mathrm{q}$ arm of the $\mathrm{X}$ chromosome. A total of 23 presumably regulatory repeats, located in CpG islands, build up a cluster at Xq23. They belong to a $53 \mathrm{~kb}$ region (chrX:114,952,840-115,006,118) with a complex structure, which reveals a number of local duplications. For this reason, all the fragments of the original probes were realigned to the reference genome to scrutinize more top alignments. As a result, 3 probes became available for each repeat within the cluster, which cross-align to repeats within this region, but not to other positions in the genome. All 23 repeats were added to the final selection of tandem repeats.

A cluster with 15 untargeted coding repeats represents members of the cancer/testis gene family 47 , also known as CT47. It is comprised of 13 nearly identical loci clustered in a $118 \mathrm{~kb}$ region at Xq24 (chrX:120,002,680-120,120,440). Following the same approach, we enriched our final selection of tandem repeats with 11 loci, each having 3 cluster-specific probes.

This approach resulted in 1837 tandem repeats. They contain 837 (83\%) presumably functional repeats on the $\mathrm{X}$ chromosome (Table 1) including repeats implicated in spinal bulbar muscular atrophy, fragile $\mathrm{X}$ and Fragile $\mathrm{X}$ E syndromes.

All probes obtained for tandem repeat capture were replicated based on the available types of probes and their GC content, as described by Duitama et al. [30] (Additional file 3: Figure S1), except that each probe with a GC content below $40 \%$ or above $70 \%$ was multiplied by 4 . The resulting probe design contains 21,386 probes with a total capture size of $0.49 \mathrm{Mb}$ and is given in the Additional file 2. It includes all the necessary information for ordering the SureSelect probes (Sheet 1), and a full description of the available probes for each target including their genomic positions, replication numbers and efficiency of a particular probe combination (Sheet 2). Each tandem repeat in the final selection is targeted by 4 to 20 probes of 1 to 4 types (Additional file 3:Figure S1).

\section{Selection of XLID cases}

Initially, we selected familial cases of ID recorded by the University Hospital of Leuven where consecutive studies, such as karyotyping, individual screening of known ID genes [4], X-array-CGH [8], or X-exome sequencing [14] did not reveal any clear pathogenic variants. The protocol was approved by the appropriate Institutional Review Board of the University Hospital of Leuven, Belgium, and informed consent was obtained from the parents of the affected patients and their healthy family members. In these families, we first checked for autosomal linkage with Merlin and for X-chromosomal segregation with Minx with default parameters (MERLIN package) using 
available STR profiles of the family members. For several families, SNP-arrays were additionally performed to confirm or disprove X-linkage. The HumanCytoSNP-12v2.1 BeadChips (Illumina) were prepared according to manufacturer's instructions, and their results were analyzed with Merlin. In two families, idiopathic ID was confirmed to be X-linked: L020 (or 5X, or MRX51) [33], L061 (or 37SX). For one more family, L084 (or 78X), a suggestive linkage interval on the $\mathrm{X}$ was obtained. In each family, an EBV-PBL cell line from the proband was available. Per family, DNA from an affected and an unaffected male were chosen for sequencing of the X-chromosomal tandem repeats: 5X20 and 5X15; 78X28 and 78X19; L061_Y and L061_S (Fig. 1). Genomic DNA samples were provided by the DNA biobank of the University Hospital of Leuven.

The affected individuals of the L020 family all present with non-syndromal ID ranging from mild to moderate as described by Claes et al. [33]. It is important to note that the $5 \mathrm{X} 9$ member of the L020 family was treated as affected, and $5 \times 16$ as unaffected. The affected individuals of the L061 family presented with non-syndromal moderate ID. Two individuals also presented with epilepsy. They were non-dysmorphic and had normal neurological examination except for individual L061_MJ, who experienced a cerebrovascular accident at the age of 47 years. Family L084 includes 3 affected males with mild to moderate non-syndromal ID. The youngest individual also presented with spastic paraplegia starting in young adulthood.

\section{Library preparation and sequencing}

DNA samples were sonicated in a Focused-ultrasonicator (Covaris) into fragments with an average size of 800 $1000 \mathrm{bp}$. Library preparation was done following the manufacturer's instructions (SureSelect Target Enrichment System for Roche 454 GS FLX and GS Junior Sequencing Platforms). SureSelect libraries were directed for SMRTbell library preparation (2 kb Template Preparation Procedure: DNA damage repair till first purification of SMRTbell templates using 0.6X AMPure PB beads) and sequenced on the Pacific Biosciences RS II machine with the P5-C3 or P6-C4 reagent kit. Each library was run on three SMRT cells. Sequencing was performed by the Genomics Core of the University Hospital of Leuven. Fastq files were obtained with a minimal requirement of 6 subreads per read of insert.

\section{Analysis of the sequencing data}

We developed a bioinformatical pipeline for retrieving information on the targeted tandem repeats from the sequencing data and their subsequent genotyping (Additional file 3: Figure S2). First, adaptor sequences were removed by trimming 32 nucleotides at both sides of a read (see Additional file 4). Alignment of the trimmed reads to the reference genome was performed using Burrows-Wheeler Aligner and Smith-Waterman alignment algorithm (BWA SW) [34]. PCR duplicates were removed with the MarkDuplicates tool (Picard tools package [35]). BEDtools [36] were used to obtain the aligned reads, which were mapped within 1000 bases from the target sites, intersected the targeted tandem repeats, or spanned full tandem repeats. A custom script (see Additional files 5 and 6) was used to estimate the number of reads spanning tandem repeats together with their 20 nt-long flanking regions, considering soft-clipped reads with several partial alignments to the flanks.

Reads of insert were further analyzed with the TSSV tool [37] with a -d option, but before that an additional step was introduced to increase specificity and speed up the analysis. For each tandem repeat a custom script (see Additional file 7) filtered those reads that were mapped within 300 bases distance from the targeted region, and created separate input files for the TSSV tool: a fasta file with filtered reads and a corresponding TSSV library. TSSV libraries included names of tandem repeats, left and right flanking sequences which were fetched from the reference genome on the Galaxy platform, and repetitive units with expected ranges of copy number (lowest and highest copy numbers set equal to the reference copy number in this case). For tandem repeats included in clusters at Xq23 and Xq24, unique cluster representatives were searched for in fasta files containing all reads mapped to the $\mathrm{X}$ chromosome.

TSSV output was processed with a custom script (see Additional file 8), which analyzes allele lengths, calculates copy numbers, implements genotyping principles described in Duitama et al. [30], and determines which copy numbers correspond to partial reads where only one of the flanking regions was found by the TSSV.

\section{Validation of sequencing derived genotypes and their inheritance in a family}

The genotypes obtained by massively parallel sequencing and data analysis were validated by fragment analysis in family members and if necessary in up to 100 controls. Control sampling comprised unaffected members of other families and patients admitted to the hospital with other, non-neurological diseases. PCR was performed in two rounds consisting of 15 and 20 cycles respectively. The first round was performed on $50 \mathrm{ng}$ genomic DNA in a $25 \mu$ l mixture using Taq DNA Polymerase (Invitrogen) and $0.2 \mu \mathrm{M}$ unlabeled specific primers designed with Primer3 [38]. All forward primers contained a $21 \mathrm{bp}$ extension of the M13 sequence at the $5^{\prime}$-end. Of the first PCR product, $2 \mu \mathrm{l}$ were used as a template for the second reaction containing a FAM-labelled M13 primer and a locus-specific reverse primer. Final products were run on 


\section{L020 (or 5X, or MRX51) family}

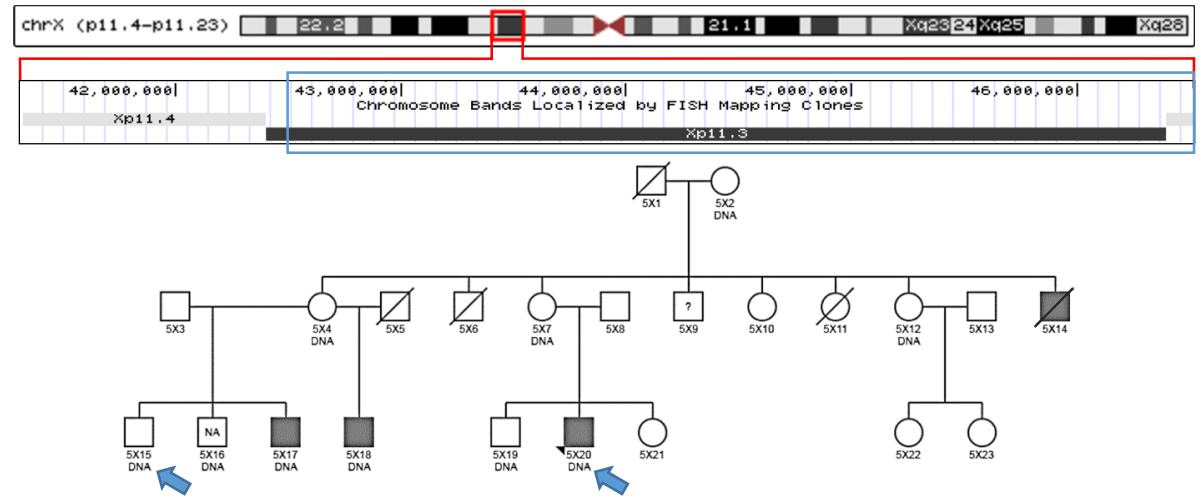

L061 (or 37SX) family

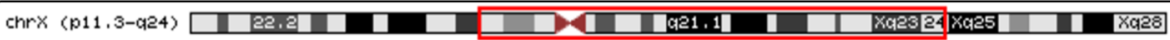

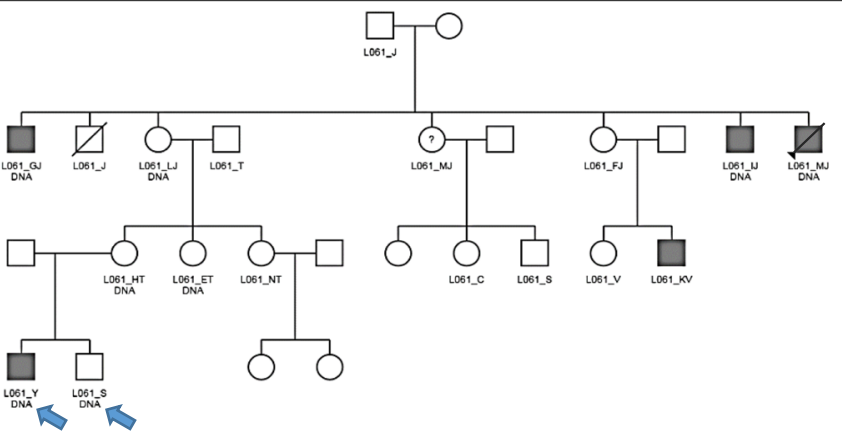

L084 (or 78X) family

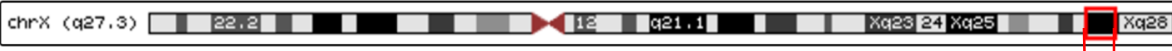

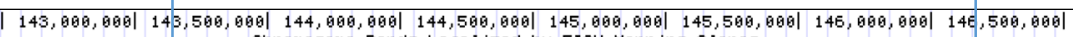
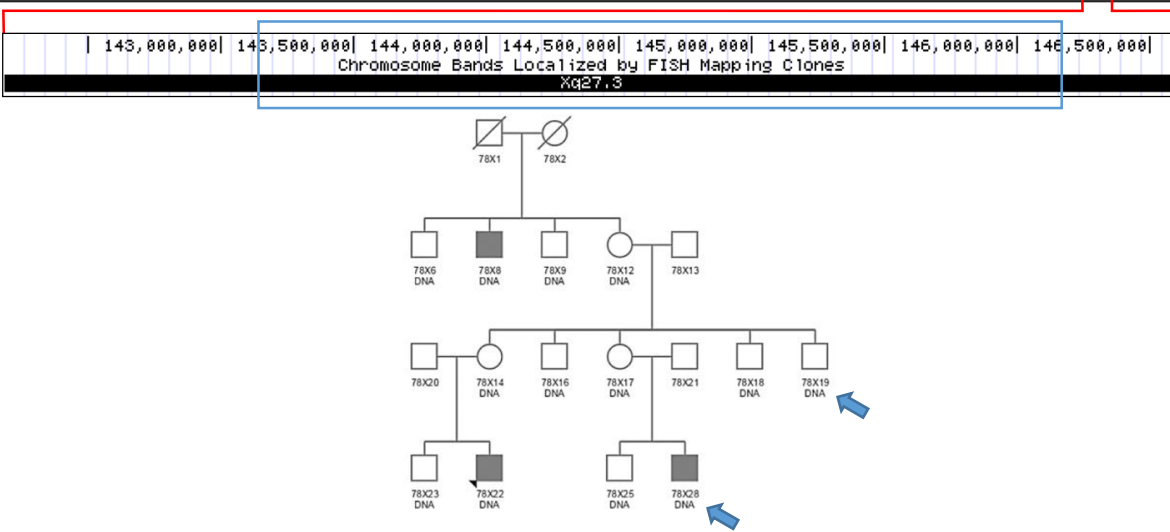

Fig. 1 Pedigrees of the selected families with idiopathic XLID. Probands are marked with a black arrow head. Grey filling indicates ID phenotype. Blue arrows point out individuals selected for targeted capture and sequencing of tandem repeats. 'DNA' stands for available genetic material. Ideogram of the X-chromosome with a zoom into the linkage interval is depicted for each family. Red and blue boxes indicate initial and refined linkage intervals, correspondingly

an ABI3500xL Genetic Analyzer (Thermofisher) with the GeneScan 500 ROX Size Standard (Thermofisher). Fragment lengths were analyzed with the GeneMapper v4.1 software (Thermofisher). For the loci possibly expanded and located within a linkage interval, we performed Sanger sequencing on the first PCR products with the respective unlabeled primers and the BigDye v3.1 cycle sequencing kit. Products were analyzed on the ABI3500xL, and resulting sequences were aligned with the BioEdit v7.1 software (Ibis Biosciences) to count the exact number of units in a tandem repeat. PCR primers used in this study are given in Additional file 9. 


\section{Quantitative PCR}

For MIR222 expression analysis RNA was extracted from EBV-PBL cell lines using mirVana miRNA Isolation Kit (Thermofisher) following small RNA enrichment procedure. RT-PCR for small RNA was performed using the TaqMan MicroRNA Reverse Transcription Kit (Thermofisher). Expression level was measured by qPCR using miRNA-specific TaqMan Small RNA Assays (Thermofisher) with 2 endogenous control miRNAs: hsa-let-7f-5p, MIR98. This was done in two independent RT-qPCR experiments. For expression analysis of other genes, total RNA was extracted from the non-confluent cell cultures using RNeasy Mini Kit (Qiagen), and cDNA was synthesized with Superscript Reverse Transcriptase and random primers (Thermofisher). Expression levels were measured 2-3 times by qPCR using SYBR Green on the LC480 apparatus (Roche) with 3 endogenous control genes: GUSB, HPRT1, PORCN. Primers used for qPCR in this study are given in Table S2, Additional file 3.

\section{Total RNA sequencing}

Total RNA was extracted from the non-confluent EBV-PBL cell cultures using RNeasy Mini Kit (Qiagen), and cDNA was synthesized with Superscript Reverse Transcriptase and random primers (Thermofisher). Total RNA single-end Illumina sequencing generated $50 \mathrm{bp}$ reads, which were mapped to the hg19 human reference genome using Tophat version 2.0.6 [39]. BAM files were handled with SAMtools version 0.1.18 [40]. Quantification of reads per gene and differential expression analysis was performed with Cufflinks version 2.0.2 [41]. Differentially expressed genes were first pre-selected with the false discovery rate of $5 \%$. To filter the most deregulated genes, they were ranked according to the ratio $(R)$ of the difference between the patient expression value $(\mathrm{P})$ and the closest control value $(\mathrm{C})$ to expression range within controls: $\mathrm{R}$ $=|\mathrm{P}-\mathrm{C}| /\left(\mathrm{C}_{\max }-\mathrm{C}_{\min }\right)$. Loci with statistically significant difference of expression $(p<0.001)$ in patient comparing to three controls were subjected to pathway enrichment analysis using IPA (Qiagen).

\section{Results}

Tandem repeat capture and sequencing in XLID families

We analyzed three families with idiopathic XLID for $\mathrm{X}$-chromosomal tandem repeat variation. In the past, in families L020, L061 and L084 neither full coverage X chromosomal microarrays, nor X-exome sequencing [4, 14] revealed any pathogenic variants. In those families, linkage analysis results in LOD scores of respectively 2.406, 2.23 and 0.932, suggesting X-linkage (see Materials and Methods). The family trees, affected and unaffected family members selected for targeted resequencing, as well as the linkage intervals are shown in Fig. 1 and Table 2. Taken that the average prevalence of intellectual disability in Western countries is $2 \%$, and the majority of cases are sporadic, we could use this frequency to estimate the probability of at least two or three causal factors (de novo mutations and/or environmental factors) co-occurring within one family: $4 \times 10^{-}$ ${ }^{4}-8 \times 10^{-6}$. Therefore, we consider a combination of several different etiologies within a single family to be unlikely, especially when the detected linkage interval on the $\mathrm{X}$ chromosome is significant (LOD $>2$ ).

For each XLID family an affected and an unaffected male was selected for targeted capture and long-read single-molecule sequencing of the tandem repeats on the X-chromosome (Fig. 1). On average, more than 135,000 reads are obtained per sample, of which $28 \%$ map within $1 \mathrm{~kb}$ from our targets, and almost $20 \%$ of the reads are useful for genotyping, as they span a target together with both flanks (Table 3). For $8.68 \%$ of the targets we could not obtain any reads, and for $1.61 \%$ of the loci we only obtained sequences that do not span the full repeat length. We obtained full sequences for 88 to $93 \%$ of the targets in the sequenced individuals with an average coverage of 10 to 23 consensus reads per locus. All obtained genotypes are given in Additional file 10.

For tandem repeats with moderate GC content (including non-functional repeats) capture and sequencing success reaches 99.0-99.6\% ( Additional file 3: Figure S3; Table 3), although for functional targets with high GC content it is $\sim 70 \%$ lower. We obtained full sequences for almost all GC-poor loci $(<40 \% \mathrm{GC})$, while

Table 2 Linkage analysis confirmed X-linkage in 3 familial cases of intellectual disability

\begin{tabular}{lllll}
\hline Family & Linkage interval & & Mbp & LOD \\
\hline L020 (or 5X, or MRX51) & Initial & chrX:41,323,975-46,534,411 & 5.21 & 2.406 \\
& Refined & chrX:42,505,938-46,534,357 & 4.03 & 57.08 \\
L061 (or 37SX) & Initial & chrX:46,179,305-103,255,350 & 9.25 & 7.23 \\
& & chrX:103,255,350-112,506,789 & 2.23 \\
& & chrX:112,516,866-120,180,324 & 4.42 & 2.23 \\
L084 (or 78X) & Initial & chrX:142,184,383-146,607,898 & 3.932 \\
& Refined & chrX:143,125,342-146,175,617 & 1.042 \\
\hline
\end{tabular}


Table 3 Sequencing yield demonstrated high recovery rate for tandem repeats in the assay

\begin{tabular}{lll}
\hline & & Yield from 3 SMRT cells \\
\hline Sequencing yield & Total consensus reads & 135,502 \\
& Unmapped & 1696 \\
& Within 1000 bases from targets & 37,608 \\
& Intersecting tandem repeats & 32,778 \\
& Spanning tandem repeats & 29,024 \\
& Useful reads (spanning tandem repeats with 20 nt flanks) & 26,855 \\
Useful reads per target & Average & $10.2-22.8$ \\
& Median & $10-23$ \\
Sequenced targets & Maximum & $63-166$ \\
& Total repeats & $88.4 \%-93.3 \%$ \\
\hline
\end{tabular}

for GC-rich regions (>70\% GC) we observe a decrease in recovery rate to $\sim 30 \%$ ( Additional file 3: Figure S3) despite the equal probe quadruplication for both groups. Efficiency of a corresponding probe combination is given for each targeted tandem repeat in the Additional file 2 (Sheet 2) together with the influence of the GC content, number of available probe types, total number of the used probes, and full length of a repetitive locus.

\section{Expansions in large tandem repeats are even detected with partial reads}

Because highly expanded alleles could exceed the size of fragments enriched in the libraries, we searched for loci that exclusively yielded reads that did not span the complete repeat. An example of such a large repeat expansion detected by this assay is an intronic repeat in the CLCN5 gene, represented by 15 copies of $26 \mathrm{bp}$ in the reference genome. We estimated copy numbers for all partial alleles in the sequenced individuals, and the longest ones correspond to at least 24-35 copies (Additional file 10). Thus, the full repeat length of the longest allele is estimated to be more than $900 \mathrm{bp}$, which is considerably longer than the reference repeat length of $390 \mathrm{bp}$. This expanded repeat is present in both affected and unaffected individuals in all three families, and since it is located outside the linkage interval in 2 families, the repeat is likely not a causal variant for XLID.

\section{XLID25 expansion in L020 is potentially linked to the phenotype}

Apart from individual repeats, we also included clustered tandem repeats in our analysis. The following strategy was used in each family to narrow down the list of candidate variants. Loci within the linkage intervals, which provided a different unit copy number in the affected versus the unaffected male were then genotyped in other family members by fragment analysis. Moreover, linkage analysis was repeated using these polymorphic repeats as additional segregation markers, allowing to refine the linkage intervals for L020 and L084 families (Table 2; Additional files 11, 12 and 13). Since we expected that mutations in repeat copy number occurred independently in these three XLID families, we checked for unique copy numbers in the patients, which segregated with the phenotype in the family and were absent in the other families. The existence of such variants in the general population was further screened for in a control sampling of up to 100 males. Only copy number variants that were not found in controls were then considered as ID candidate loci.

For the L084 family, 18 tandem repeats were targeted by our assay in the linkage interval, of which 17 are successfully sequenced. Of those, only one intergenic tandem repeat at chrX:145,340,826-145,341,025 (XLID32) exhibits a copy number difference between the affected (78X19) and unaffected (78X28) individuals. However, this allele is also found in control samples and thus considered to be a benign variant.

For the L061 family, we obtained 315 presumably functional loci within the linkage interval with a coverage of at least 5X. Forty five of these repeats were found to be polymorphic within the normal variation range obtained in other XLID families; fragment analysis demonstrated that 3 variants (XLID75, XLID77, XLID79) were false positives; 1 other variant (XLID76) was in the normal size range when compared to additional controls (Table 4); and for 4 repetitive loci (XLID73, XLID74, XLID78, XLID80) the apparent unique copy number detected only in the affected males of the L061 family were also found in the unaffected control population (Table 4). Finally, the tandem repeat XLID72, which displays a shorter polyglutamine tract in the proband compared to his unaffected relative and other families, is located in the first exon of the well characterized $A R$ gene. Since the array length of the 


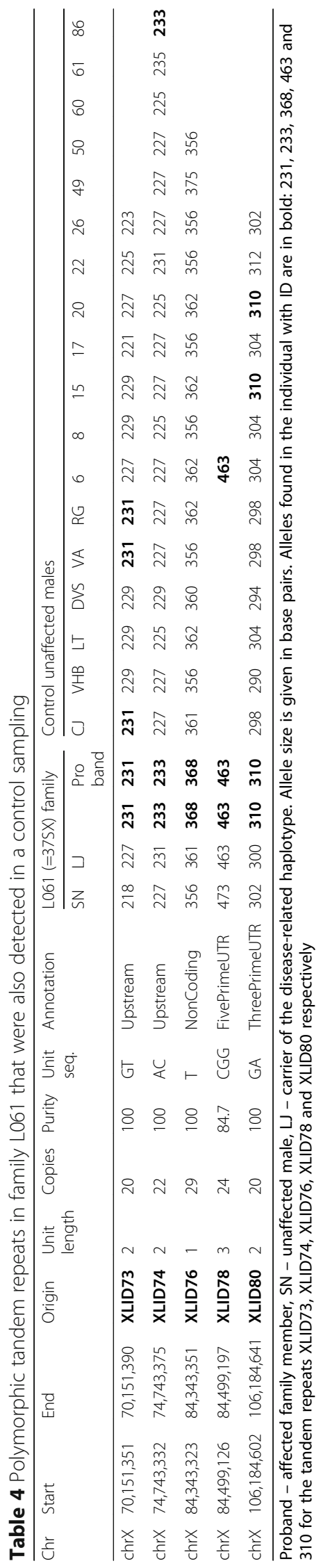


tandem repeat chrX:66,765,149-66,765,262 is within the normal range, it is considered to be not related to ID.

For the L020 family, 44 targeted tandem repeats are present in the linkage region, of which 43 are successfully sequenced. Of these, 5 repeats located in regulatory regions exhibit a copy number difference between the affected (5X20) and unaffected (5X15) family members. However, following fragment analysis, in 4 loci (XLID2, XLID20, XLID22, XLID27) alleles detected in 5X20 and his affected relatives were also detected in 20 control samples (Table 5).

The tandem repeat at chrX:45,606,270-45,606,355 (XLID25), which reveals a unique allele in the affected family members segregating with the phenotype, is located 65 bp downstream of the microRNA gene MIR222. It is a complex repeat consisting of two consecutive $(\mathrm{CT})_{\mathrm{n}}$ and $(\mathrm{GT})_{\mathrm{n}}$ sub-repeats separated by three thymidines (Fig. 2). After screening the entire control population of 100 healthy males by fragment analysis only two exhibited the same total length as in the L020 proband. We performed an additional screening by fragment analysis to differentiate $(\mathrm{CT}) \mathrm{n}$ and (GT)n sub-repeats in those controls (Table S3). For that, we used a nested PCR primer annealing to the boundary that separates the sub-repeats, which gives an approximate estimation of CT copy number. To define the exact length of each individual repeat, amplicons of the 5X17 proband along with that of 23 control males were Sanger sequenced (Fig. 2). The sequenced controls included the samples with the estimated CT copy number equal to or one copy shorter than that of the proband, and the samples with the allele of the same total length as the proband. The latter control samples demonstrated different copy numbers for both $(\mathrm{CT})_{\mathrm{n}}$ and $(\mathrm{GT})_{\mathrm{n}}$ sub-repeats $(17 \mathrm{CT}$ and 37 GT copies or $20 \mathrm{CT}$ and 34 GT copies), compared to the proband who exhibited an allele with the highest CT copy number (21), while the GT unit number (33) was in the normal range of 24-37 copies, observed in the controls. Notably, none of the sequenced samples revealed the $(\mathrm{CT})_{\mathrm{n}}$ sub-repeat longer than 20 copies.

\section{Upstream MIR222 gene reveals decreased expression, and} its targets FMR1, NEFH are up-regulated in the proband

To investigate the possible effect of the XLID25 CT-sub-repeat expansion on MIR222 expression, we performed a miRNA specific TaqMan assay on the enriched small-RNA samples extracted from EBV-PBL cell lines of the proband and three male controls. As no cell lines were available for the other family members, their MIR222 expression has not been tested. The results show that MIR222 expression is decreased at least 5 -fold in the patient (Fig. 3a) compared to the control samples that correspond to 'Co14' (genotype $17 \mathrm{CT}, 32 \mathrm{GT}$ ), 'Co15' (17 CT, 32GT) and 'Co17' (17 CT, 35GT) males in the sequence alignment in Fig. 2. Additionally, we tested the expression of one of the MIR222 downstream targets, $F M R 1$, known to be involved in intellectual disability. Interestingly, the FMR1 mRNA levels are elevated by $30 \%$ in the XLID patient compared to the 3 controls, who show highly similar levels (Fig. $3 \mathrm{~b}$ ).

To reveal additional deregulated genes that might be affected by the altered MIR222 expression, we performed RNA sequencing again on RNA extracted from EBV-PBLs of the patient and 3 controls, same as in the previous experiment. We obtained 46 loci that are significantly and consistently up- or down-regulated in the patient, however none of them is within the linkage interval of the family. Based on Ingenuity Pathway Analysis most of these genes are involved in anatomical structure morphogenesis, cellular component movement, locomotion and localization of the cell. $\mathrm{R}$ ratio $\geq$ 0.9 for expression deregulation in patient is observed in 35 genes, of which 31 are known to be expressed in brain. Of these, 21 genes $(68 \%)$ are predicted targets of MIR222, while it is expected to regulate half as many (30\%) in an entire pool of the brain-expressed genes. Nine genes were selected to confirm the RNAseq data by qPCR. Upon increasing the number of controls to 7 , the altered expression in the patient remained apparent in 2 of the 9 selected genes: ARMCX2 and NEFH (Fig. 4), which are predicted targets of MIR222 (microRNA.org). Notably, the NEFH gene encoding the heavy neurofilament protein reveals a 52-fold increased expression level in the XLID patient in relation to the 7 controls. As for the genes within the linkage interval other than MIR222, for 7 of them (MAOA, DUSP21, PPP1R2P9, MAOB, NDP, EFHC2 and MIR221) we did not obtain any RNA sequencing data while other 8 (KDM6A, ZNF673, FUNDC1, CXorf36, ZNF674, KRBOX4, ZNF674-AS1 and CHST7) reveal expression differences that are not statistically significant ( $p$-values $0.29-0.99$ ).

\section{Discussion}

Despite multiple large-scale array-CGH and exome and genome sequencing analyses, the genetic variation underlying X-linked intellectual disabilities remains elusive for a large number of families. We hypothesize that tandem repeat expansions have escaped detection mainly due to short-read sequencing technologies. Here, we developed an extensive screen for $\mathrm{X}$ linked tandem repeat expansions using a long read MPS approach. We captured and sequenced more than 1800 tandem repeats in three families with idiopathic XLID and demonstrate the feasibility of single molecule sequencing to accurately detect and size tandem repeats and tandem repeat variability. Moreover, in one family a tandem repeat expansion segregating with ID seems to affect the expression of the nearby MIR222 gene, previously implicated in ID 


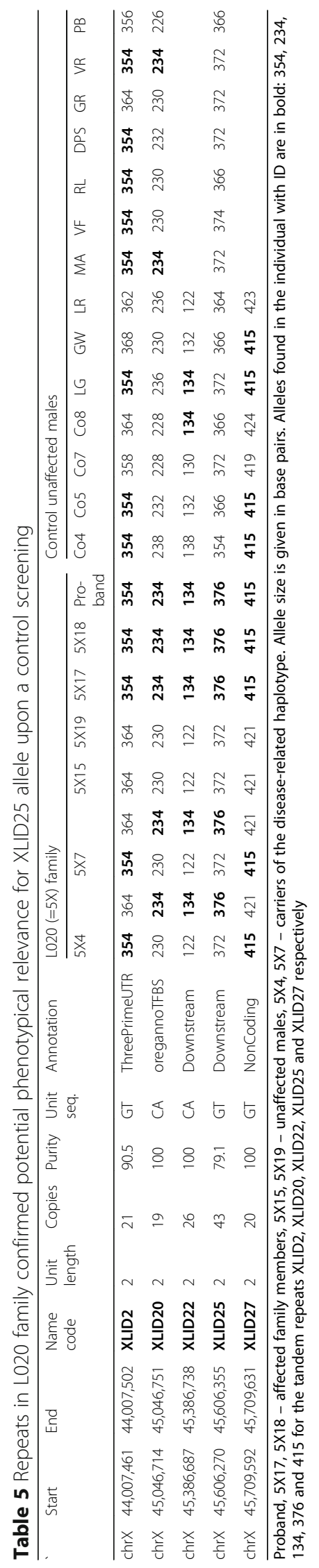




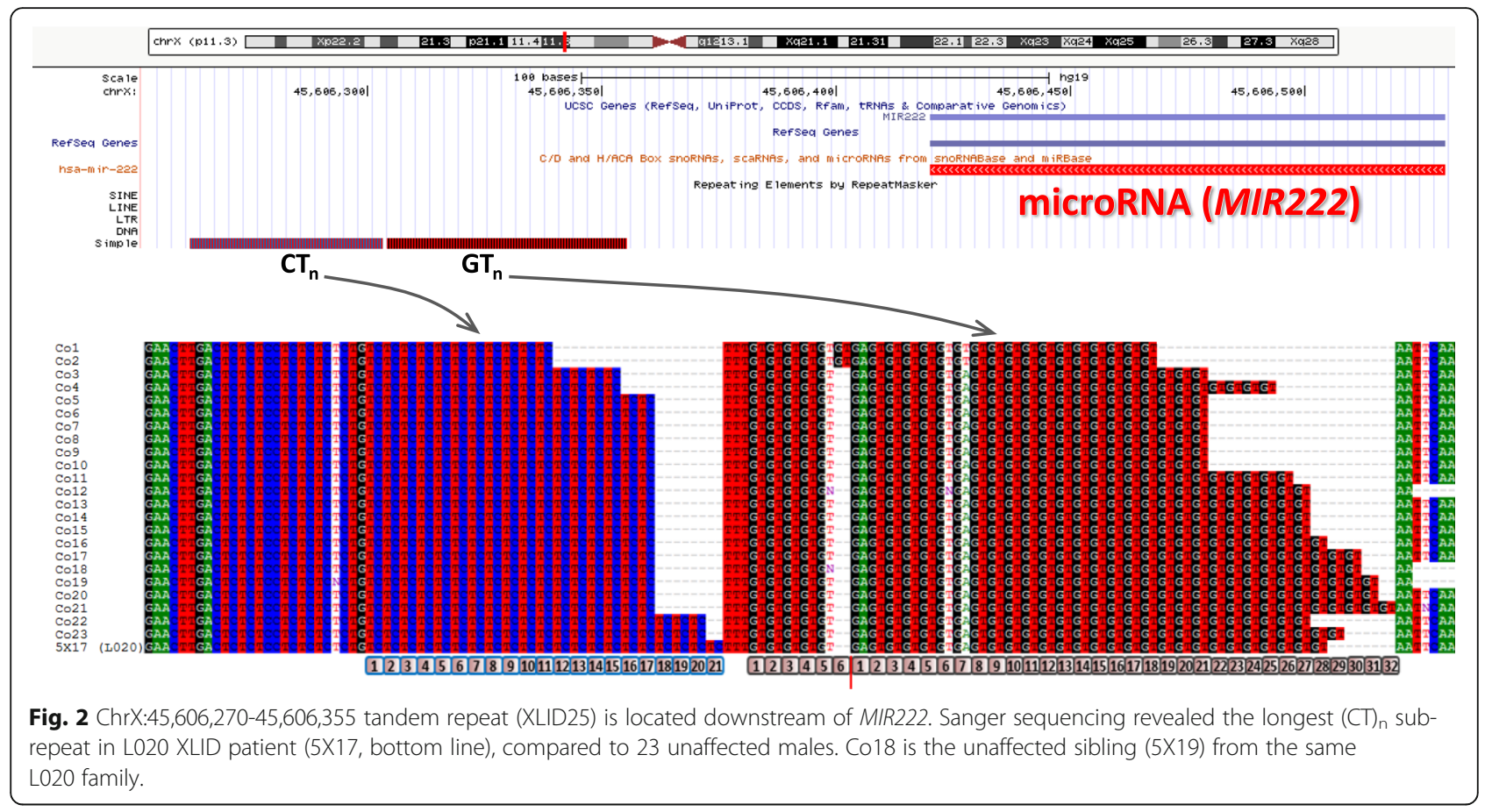

[42] and affecting expression levels of genes associated with ID [43-46].

Multiple studies have been reported on large-scale analysis of tandem repeat polymorphism in several species, with various enrichment strategies, and utilizing different massively-parallel sequencing technologies. Information on thousands of repeats was previously retrieved from whole-genome or targeted sequencing data for 8 individual genomes [47], 550 and later 1009 individuals from the 1000 Genomes Project [48, 49], or 34 human gastric cancer cell lines and tissue samples [50]. An extensive characterization of $>390$ thousand loci in more than 150 Drosophila strains by Fondon et al. [51] provided an important framework for further association studies in this species. However, Illumina read length limits target loci to microsatellites shorter than $\sim 90 \mathrm{bp}$ or less. On the contrary, the use of the 454 platform, generating longer reads, allowed to discover hundreds to thousands of new microsatellites in 14 species among animal, plant and fungi taxa [52], or selectively target human tandem repeats with a broader range of characteristics [30] with a total length up to $\sim 300 \mathrm{bp}$. More recently, long read
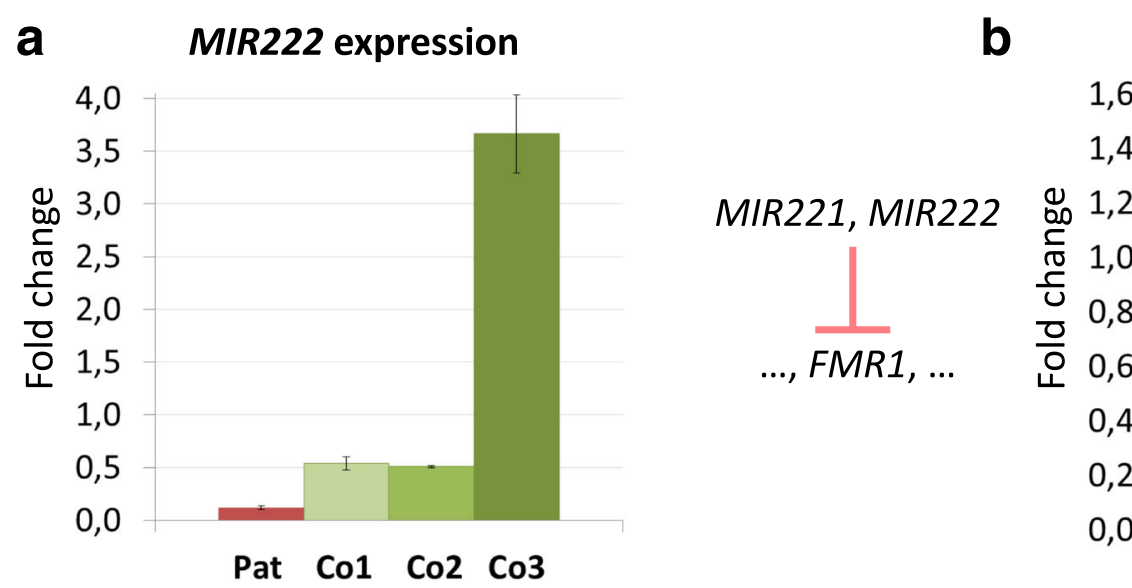

FMR1 expression

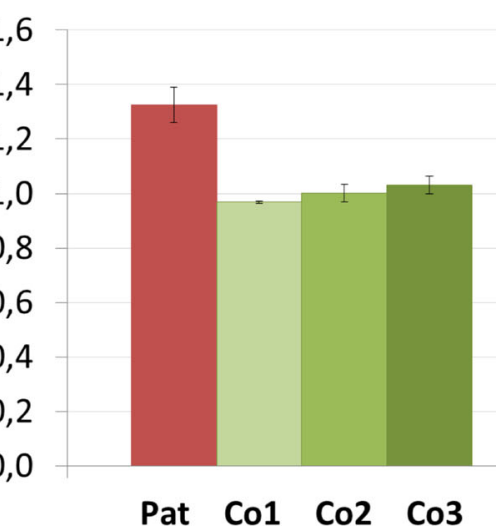

Fig. 3 The expression level of MIR222 (a) is decreased while one of its targets, FMR1 (b), is up-regulated in the patient ('Pat', red) opposed to 3 controls ('Co1', 'Co2', 'Co3', shades of green). Error bars show standard deviation of the normalized expression in 2 and 3 experiments, respectively. 'Co1', 'Co2', 'Co3' samples correspond to 'Co14' (17 CT, 32GT), 'Co15' (17 CT, 32GT), 'Co17' (17 CT, 35GT) males in the sequence alignment in Fig. 2 

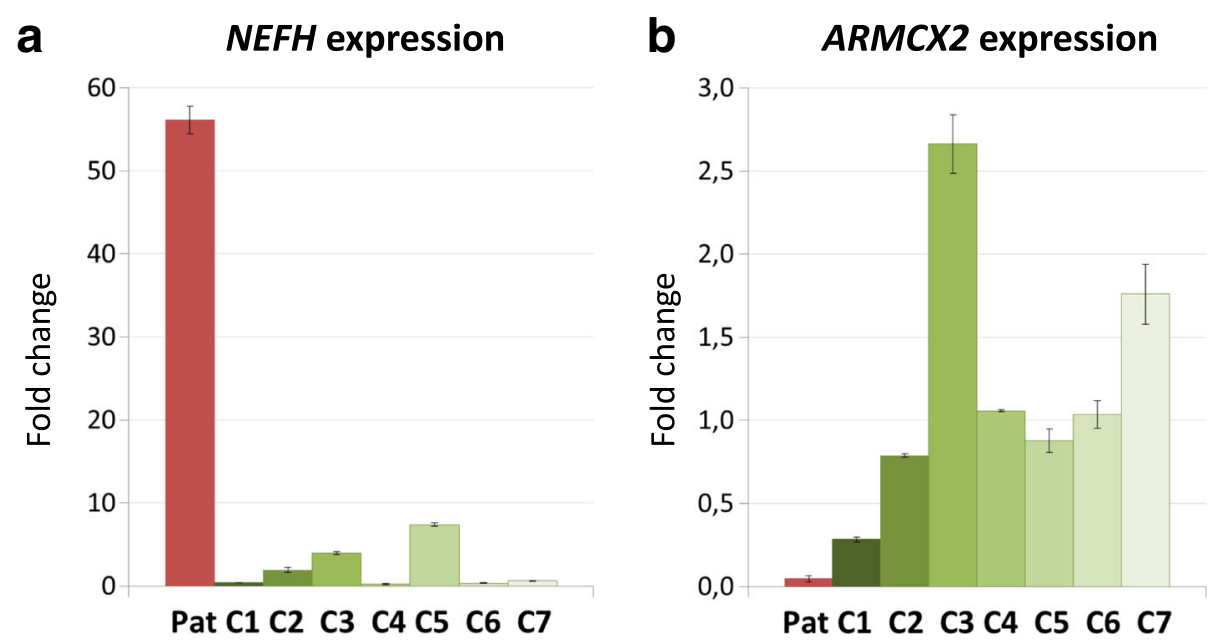

Fig. $\mathbf{4}$ Up-regulated expression of NEFH (a) and down-regulation of ARMCX2 mRNA (b) in the affected proband ('Pat', red) and 7 controls ('C1-7', shades of green). Error bars show standard deviation of the normalized expression in 3 experiments

single molecule sequencing has been introduced to study tandem repeats, though limited to only a few loci so far [21-23]. Here, we combined the benefits of broad-range targeted sequencing with the large read size, which permitted analysis of virtually all coding and regulatory repeats on the $\mathrm{X}$ chromosome. Overall, $83 \%$ of all likely functional repeats could be targeted with unique probes, and on average more than $90 \%$ of targets were captured and sequenced.

Capture-based enrichment has previously been applied in studies of tandem repeat variation. The first approach [52] used 8 spanning probes designed to bind all microsatellites in the genome with probe-matching motifs. Later studies focused on a more selective and specific capture approach using probes complementary to the unique flanking regions of such repeats, which boosted the capture efficiency. Guilmatre et al. [47] utilized solely flanking probes and increased the on-target sequences to $39 \%$. The design of longer flanking probes, as well as the addition of spanning and special double-flank probes that we previously described even raised the capture efficiency to $62 \%$ [30]. Our current probe design, based on the latter approach, however, resulted in a lower on-target rate of $28 \%$. This apparent capture efficiency drop is likely due to a more than 5 -time decrease in the number of targets compared to Duitama et al. [30]. According to the manufacturer (personal communication), this is a frequently observed effect of excess baits that are forced to bind aspecifically when there are not enough target molecules for a proper hybridization. Hence, it was suggested to test half of the recommended bait amount for the future hybridizations. Another difference in the probe design that probably has a minor effect is the ratio of the probes with extreme GC content. A higher percentage of probes with low GC content is likely to hybridize non-specifically to AT-rich Alu elements that are abundantly present in the genome, thereby increasing the off-target count. However, despite the large off-target rate our assay demonstrated high recovery rate of the targeted regions. It is clear though that optimizing the baits stoichiometry and further improvements to the probe design have the potential to boost the capture efficiency even more.

Interestingly, tandem repeat expansions can be detected even if the expansion exceeds the targeted fragment length. As an example, we detected expansion of more than 900 bp for an intronic tandem repeat in CLCN5 that is only $390 \mathrm{bp}$ long in the reference genome. This expansion would not be detected with short-read technologies. We found such elongated alleles in all six sequenced individuals suggesting either a significant length variation in the population, or a local misassembly and a hidden gap in the reference genome. If so, this would not be surprising considering that multiple studies find repeat arrays in remaining gaps of genome assemblies [53-55].

A series of tandem repeats were shown to be variable in length between affected and unaffected relatives. However, all but one did not seem to be associated with ID as they were also found in healthy controls or were in disagreement with the clinical description. In one of the families (L020), a single repeat ('XLID25') consisting of two adjacent tandem repeat stretches revealed a unique expanded allele of 21 copies in the CT sub-repeat in the proband, whereas all other genotyped healthy individuals of the family as well as controls had unit numbers in the range of 11-20 copies.

CT-repeats (or GA-repeats in complement), also known as GAGA-elements, act as chromatin remodelling mediators by disrupting and displacing pre-assembled nucleosomes [56-58]. Emamalizadeh and colleagues [59] 
suggested that the $(\mathrm{GA})_{11}$ repeat length in the promoter of RIT2 is crucial for obtaining the correct dosage of RIT2, important in regulating the neuronal function. A shorter allele $(G A)_{5}$ in homozygous state has been detected only in a proband with schizophrenia thus linking it to the disease state. Similarly, length variation of $(\mathrm{GA})_{\mathrm{n}}$ tandem repeats influences embryonic development and facilitates evolutionary adaptation by regulating $M E C O M$ and GABRA3 expression [60]. It has been reported that GAGA-binding protein in humans specifically binds to the elements of 8 GA-units [61], which explains why precise copy number ranges are extremely important in regulatory sites. GA-dinucleotides may also affect gene expression when located downstream of that gene [62], which is in agreement with our data.

Only 65 bp upstream of XLID25 a highly conserved microRNA gene, MIR222, is located. MIR222 is mainly expressed in telencephalon with a conserved pattern of expression in larval and adult brain in zebrafish [63]. The MIR221/222 cluster is known to play an important role in coordination of cell proliferation [64]. They were shown to regulate terminal differentiation of neurons in porcine cortex and cerebellum [65]. Moreover, MIR222 was demonstrated to regulate timing of neural development by blocking preliminary generation of bipolar neurons in Xenopus [66]. It has been suggested that MIR222, with or without MIR221, is a plausible candidate to cause intellectual disability associated with X-linked retinal dystrophy in the Xp11.3 deletion syndrome [42]. Interestingly, in a study by Chen et al. [67], screening of 13 brain-expressed miRNAs in 464 patients with X-linked intellectual disability revealed only 4 mutations, of which 2 segregated with the phenotype, and both were found in MIR222. One of these mutations was located near the Drosha ribonuclease cleavage site and therefore, could potentially affect mature miRNA formation. On the other hand, the high conservation of the brain-expressed MIR222 suggests an important function in this tissue. We demonstrated that in the proband of the L020 family expression levels of MIR222 were decreased. In consonance with our study, MIR222 and MIR221 levels were previously found to be down-regulated in hippocampal tissue of patients with a neurological disease - mesial temporal lobe epilepsy and hippocampal sclerosis [68]. All these studies point to a crucial role of MIR222 for proper brain functioning. Decreased levels seem to disturb yet enigmatic neuronal processes.

We also tested the expression of one of the MIR222 targets namely FMR1. FMRP is an mRNA binding protein that has multiple functions in post-transcription gene regulation including mRNA stability, mRNA transport and localization, translation control, and pre-mRNA alternative splicing [69] with the latter being more prevalent in brain compared to other tissues [70]. FMR1 is usually the primary gene to test in the newly diagnosed ID patients [4]. Large tandem repeat expansions cause silencing of this gene that leads to intellectual disability with more severe forms in males. Contrary to this most common mechanism, we demonstrated a significantly increased expression of FMR1 in the affected male of L020. This controversy may suggest that precise concentration of FMRP in the brain is required for its proper function, and any dysregulation disrupting the balance can cause a disease. Our data is in line with the fact that the gene was shown to cause abnormal behavior when overexpressed in mice, a specific high-anxiety phenotype that is different from FMR1 knock-out mice [71]. Elevated FMR1 expression levels were also suggested to be causal in 5 ID patients carrying a duplication that harbors the FMR1 gene, amongst several others [43-46]. However, only one study [44] looked into mRNA expression in blood of a proband, which was within the normal range. Either the presence of two copies in males is not sufficient to cause overexpression or the expression levels in brain are different from those in blood cells leading to tissue-specific consequences.

In order to detect additional genes that could be regulated by MIR222, we performed total RNA sequencing and detected a second deregulated target of MIR222 namely $N E F H$. Its product is a component of neural cytoskeleton important for neuronal maintenance and plasticity, neurite outgrowth, axonal caliber and transport [72]. Interestingly, in the L020 proband this gene was 50 -fold up-regulated. As shown by Collard et al. [73] overexpression of human NEFH in mice causes defects in axonal transport, which eventually leads to neuron degeneration. Notably, NEFH protein was described 1.5 -fold up-regulated in children with cortical dysplasia with epilepsy [74].

All these findings provide indirect evidence that the unique tandem repeat variant of 21 copies of the CT/ GA repeat is a strong candidate for the ID phenotype in the L020 family. The expanded repeat might cause decreased expression of the nearby MIR222 microRNA resulting in a decreased breakdown of neuronal target genes including FMR1 and NEFH.

To confirm a causal link between the detected tandem repeat variant and the XLID phenotype, further studies are required. Future screening of affected cohorts and healthy population for XLID25 expansions, microRNA MIR222 mutations, expression profiles of MIR222 might reveal more cases and statistical significant associations. In addition, cellular experiments will assess the impact of the repeat size on the MIR222 expression in the same patient cell line. Potentially, CRISPR/Cas9-induced double-strand break within the tandem repeat will lead to the reparation-induced repeat instability $[75,76]$. This should allow to generate cell lines with a common genetic background and only different by the repeat copy 
number which would provide a reliable functional model. Finally, a knock-out of the microRNA in a mouse model would demonstrate its role in the XLID development.

To our knowledge, this is the first study capturing and single molecule sequencing targeted genomic loci. This strategy can be applied to other targets as well as to repeats elsewhere in the genome. Though this particular probe design is only applicable to the X-linked disorders, our tandem repeats screening approach is expandable to other chromosomes. With time, improved genome annotation might require an update of the list of tandem repeats that are potentially relevant for a disease development.

Whereas our method allows the detection of a majority of repeat expansions, it might fail on longer repeats. In this study, we targeted repeats up to $500 \mathrm{bp}$. This enabled sequencing over the repeat multiple times, generating proper consensus lengths. To accurately measure repeat lengths, the polymerase reads must be at least six times the size of the insert. With a mean polymerase read length of $15 \mathrm{~kb}$, the repeat sizes should have a maximum length of $2-2.5 \mathrm{~kb}$. Nevertheless, with ever expanding longer reads, the repeats sizes that can be measured, will also be expanded. However, such long repeats constitute only a small portion of all repeats in the genome. A second limitation is that the capture method has a reduced performance on GC-rich tandem repeats or fragments. Although the single molecule sequencing has no problem passing GC-rich repeats, those sequences show reduced capture efficiency.

\section{Conclusions}

Our findings provide indirect evidence that the unique tandem repeat variant of 21 copies of the CT/GA repeat is a strong candidate for the ID phenotype in one of the studied families with X-linked ID. The expanded repeat might cause decreased expression of the nearby MIR222 microRNA resulting in a decreased breakdown of neuronal target genes including FMR1 and NEFH. Present work is the first large-scale study of targeted sequencing of tandem repeats as a means to improve diagnosis of an inherited disease. Future application of the described assay in a large number of cases will allow to evaluate the general contribution of tandem repeat instability to XLID. Next to XLID, our design may be used to study other X chromosome related diseases too. Moreover, this approach is not restricted to the $\mathrm{X}$ chromosome, but is applicable to screen for tandem repeats on other chromosomes as well.

\section{Additional files}

Additional file 1: Custom script searching for unique capture probes that are closest to the target within a specified distance. ( $\mathrm{SH} 13 \mathrm{~kb}$ )
Additional file 2: SureSelect capture probe design and information on the performance of the different probe combinations per target. (XLSX $1353 \mathrm{~kb})$

Additional file 3: Tables S1-S2, supplemental Figures S1-S3, sample tables of the Additional files 2, 9 and 10. (PDF $810 \mathrm{~kb}$ )

Additional file 4: Custom script removing adaptor sequences from the sequencing reads. (PY $1 \mathrm{~kb}$ )

Additional file 5: Custom script for estimating the number of reads spanning a tandem repeat together with its 20 nt-long flanking regions, considering soft-clipped reads with several partial alignments to the flanks. (SH 2 kb)

Additional file 6: Supplementary script called by the countTargetSpanning.sh script (Additional file 5). (PY 2 kb)

Additional file 7: Custom script filtering the reads that were mapped within a specified distance from the targeted region, and creating a separate input file for the TSSV tool. (PY $11 \mathrm{~kb}$ )

Additional file 8: Custom script processing the output of the TSSV tool and estimating the genotype of a tandem repeat. (PY $30 \mathrm{~kb}$ )

Additional file 9: List of primers used in this study. (XLSX $21 \mathrm{~kb})$

Additional file 10: Genotypes obtained from the sequencing data of the targeted tandem repeats in three families with $\mathrm{X}$-linked intellectual disability. (XLSX $2711 \mathrm{~kb}$ )

Additional file 11: Output data from the linkage analysis in the L020 family with $X$-linked intellectual disability. (LOG 4 kb)

Additional file 12: Output data from the linkage analysis in the L061 family with X-linked intellectual disability. (LOG 13992 kb)

Additional file 13: Output data from the linkage analysis in the L084 family with $\mathrm{X}$-linked intellectual disability. (LOG $4 \mathrm{~kb}$ )

\section{Abbreviations}

aCGH: array-based comparative genomic hybridization; EBV-PBL: Peripheral blood lymphocytes transformed with Epstein Barr Virus; GC: Guaninecytosine; ID: Intellectual disability; LOD: Logarithm (base 10) of odds; miRNA: microRNA; MPS: Massively-parallel sequencing; SMRT: Single molecule real-time; XLID: X chromosome-linked intellectual disability

\section{Acknowledgements}

We are grateful to the patients and their families for their cooperation. We would like to thank Wim Meert (Genomics Core, University Hospitals Leuven) for PacBio sequencing and troubleshooting and Greet Peeters for the help with library preparations.

\section{Funding}

This work was supported by a research grant G.0795.11 from the Fund for Scientific Research-Flanders (FWO-Vlaanderen) to KJV, GF and JRV, and a scholarship grant awarded by the Support Fund Marguerite-Marie Delacroix (Fonds de Soutien Marguerite-Marie Delacroix) to AZ, by grants from the KU Leuven PFV/10/016 SymBioSys to JRV and GOA/12/015 to JRV and HVE, the Herculesfoundation (ZW11-14) to JRV, and Belgian Science Policy Office Interuniversity Attraction Poles (BELSPO-IAP) program through the project IAP P7/43-BeMGI. The funders had no role in study design, data collection, analysis and interpretation, decision to publish, or preparation of the manuscript. HVE is a clinical investigator of the Fund for Scientific Research-Flanders (FWO-Vlaanderen).

\section{Availability of data and materials}

The SNP array and RNA sequencing datasets supporting the conclusions of this article are available in the ArrayExpress repository at EMBL-EBI (https://www.ebi.ac.uk/arrayexpress/experiments/E-MTAB-5903/ and https://www.ebi.ac.uk/ arrayexpress/experiments/E-MTAB-5904/, respectively). The SMRT sequencing dataset from this study is available at the European Nucleotide Archive, project PRJEB21596 (https://www.ebi.ac.uk/ena/data/view/PRJEB21596): accession numbers ERS1814531, ERS1814640, ERS1814637, ERS1814681, ERS1814590, ERS1814619, ERS1814620. 


\section{Authors' contributions}

GF, KJV and JRV conceived and designed the study. GF and JRV supervised the experiments. HVE provided the samples and clinical background for the studied families. AZ performed all the wet-lab experiments and subsequent data analysis if not stated otherwise, and was a major contributor in writing the manuscript. GF, KJV, HVE and JRV edited the paper. All authors read and approved the final manuscript.

\section{Ethics approval and consent to participate}

The protocol was approved by the appropriate Institutional Review Board of the University Hospital of Leuven, Belgium, and a verbal informed consent was obtained from the parents of the affected patients and their healthy family members.

\section{Consent for publication}

Not applicable.

\section{Competing interests}

The authors declare that they have no competing interests.

\section{Publisher's Note}

Springer Nature remains neutral with regard to jurisdictional claims in published maps and institutional affiliations.

\section{Author details}

'Department of Human Genetics and Center for Human Genetics, Laboratory for Cytogenetics and Genome Research, University Hospitals Leuven, KU Leuven, O\&N I Herestraat 49 - box 606, 3000 Leuven, Belgium. ${ }^{2}$ Department of Human Genetics and Center for Human Genetics, Laboratory for Genetics of Cognition, University Hospitals Leuven, KU Leuven, O\&N I Herestraat 49 box 606, 3000 Leuven, Belgium. ${ }^{3}$ VIB Center for Microbiology and CMPG Lab for Genetics and Genomics, KU Leuven, Gaston Geenslaan 1 - box 2471, 3001 Leuven, Belgium. ${ }^{4}$ Clinical Biology, Laboratory for Molecular Diagnostics, Jessa Hospital, Stadsomvaart 11, 3500 Hasselt, Belgium.

Received: 7 May 2018 Accepted: 6 December 2018 Published online: 19 December 2018

\section{References}

1. Ropers HH. Genetics of intellectual disability. Curr Opin Genet Dev. 2008;18: 241-50.

2. Gécz J, Shoubridge C, Corbett M. The genetic landscape of intellectual disability arising from chromosome X. Trends Genet. 2009;25:308-16.

3. Kleefstra T, Yntema H, Oudakker A, Banning M, Kalscheuer V, Chelly J, Moraine C, Ropers H, Fryns J, Janssen I. Zinc finger 81 (ZNF81) mutations associated with X-linked mental retardation. J Med Genet. 2004;41(5):394-9.

4. de Brouwer AP, Yntema HG, Kleefstra T, Lugtenberg D, Oudakker AR, de Vries BB, van Bokhoven $\mathrm{H}$, Van Esch $\mathrm{H}$, Frints SG, Froyen $\mathrm{G}$, et al. Mutation frequencies of $X$-linked mental retardation genes in families from the EuroMRX consortium. Hum Mutat. 2007;28:207-8.

5. Kousoulidou L, Parkel S, Zilina O, Palta P, Puusepp H, Remm M, Turner G, Boyle J, Van Bokhoven $\mathrm{H}$, de Brouwer $\mathrm{A}$, et al. Screening of 20 patients with X-linked mental retardation using chromosome X-specific array-MAPH. Eur J Med Genet. 2007;50:399-410

6. Jensen LR, Chen W, Moser B, Lipkowitz B, Schroeder C, Musante L, Tzschach A, Kalscheuer VM, Meloni I, Raynaud M. Hybridisation-based resequencing of 17 X-linked intellectual disability genes in 135 patients reveals novel mutations in ATRX, SLC6A8 and PQBP1. Eur J Hum Genet. 2011;19(6):717-20.

7. Van Esch H, Bauters M, Ignatius J, Jansen M, Raynaud M, Hollanders K, Lugtenberg D, Bienvenu T, Jensen LR, Gecz J, et al. Duplication of the MECP2 region is a frequent cause of severe mental retardation and progressive neurological symptoms in males. Am J Hum Genet. 2005;77:442-53.

8. Froyen $G$, Van Esch H, Bauters M, Hollanders K, Frints SG, Vermeesch JR, Devriendt K, Fryns JP, Marynen P. Detection of genomic copy number changes in patients with idiopathic mental retardation by high-resolution $X$ array-CGH: important role for increased gene dosage of XLMR genes. Hum Mutat. 2007;28:1034-42.

9. Bashiardes S, Kousoulidou L, Van Bokhoven H, Ropers HH, Chelly J, Moraine C, de Brouwer AP, Van Esch H, Froyen G, Patsalis PC. A new chromosome $x$ exon-specific microarray platform for screening of patients with X-linked disorders. J Mol Diagn. 2009;11:562-8.
10. Isrie M, Froyen G, Devriendt K, de Ravel T, Fryns JP, Vermeesch JR, Van Esch H. Sporadic male patients with intellectual disability: contribution of X-chromosome copy number variants. Eur J Med Genet. 2012;55(11): $577-85$.

11. Hu H, Wrogemann K, Kalscheuer V, Tzschach A, Richard H, Haas SA, Menzel C, Bienek M, Froyen G, Raynaud M, et al. Mutation screening in 86 known Xlinked mental retardation genes by droplet-based multiplex PCR and massive parallel sequencing. HUGO J. 2009;3(1-4):83.

12. de Ligt J, Willemsen MH, van Bon BWM, Kleefstra T, Yntema HG, Kroes T, Vulto-van Silfhout AT, Koolen DA, de Vries P, Gilissen C, et al. Diagnostic exome sequencing in persons with severe intellectual disability. N Engl J Med. 2012;367:1921-9.

13. Gilissen C, Hehir-Kwa JY, Thung DT, van de Vorst M, van Bon BW, Willemsen MH, Kwint M, Janssen IM, Hoischen A, Schenck A, et al. Genome sequencing identifies major causes of severe intellectual disability. Nature. 2014;511(7509):344-7.

14. Hu H, Haas SA, Chelly J, Van Esch H, Raynaud M, de Brouwer APM, Weinert S, Froyen G, Frints SGM, Laumonnier F, et al. X-exome sequencing of 405 unresolved families identifies seven novel intellectual disability genes. Mol Psychiatry. 2016;21:133-48.

15. Tarpey PS, Smith R, Pleasance E, Whibley A, Edkins S, Hardy C, O'Meara S, Latimer C, Dicks E, Menzies A, et al. A systematic, large-scale resequencing screen of X-chromosome coding exons in mental retardation. Nat Genet. 2009:41:535-43.

16. Huang L, Jolly LA, Willis-Owen S, Gardner A, Kumar R, Douglas E, Shoubridge C, Wieczorek D, Tzschach A, Cohen M, et al. A Noncoding, regulatory mutation implicates HCFC1 in nonsyndromic intellectual disability. Am J Hum Genet. 2012;91(4):694-702.

17. Legendre M, Pochet N, Pak T, Verstrepen KJ. Sequence-based estimation of minisatellite and microsatellite repeat variability. Genome Res. 2007; $17: 1787-96$

18. Vinces $M D$, Legendre $M$, Caldara $M$, Hagihara $M$, Verstrepen $K J$. Unstable tandem repeats in promoters confer transcriptional evolvability. Science. 2009:324:1213-6.

19. Gemayel R, Vinces MD, Legendre M, Verstrepen KJ. Variable tandem repeats accelerate evolution of coding and regulatory sequences. Annu Rev Genet. 2010;44:445-77.

20. Bilgin Sonay $T$, Carvalho T, Robinson MD, Greminger MP, Krützen M, Comas D, Highnam G, Mittelman D, Sharp A, Marques-Bonet T, et al. Tandem repeat variation in human and great ape populations and its impact on gene expression divergence. Genome Res. 2015;25(11):1591-9.

21. Guo $X$, Zheng $S$, Dang $H$, Pace RG, Stonebraker JR, Jones CD, Boellmann $F$, Yuan G, Haridass P, Fedrigo O, et al. Genome reference and Sequence variation in the large repetitive central exon of human MUC5AC. Am J Respir Cell Mol Biol. 2014:50(1):223-32.

22. McFarland KN, Liu J, Landrian I, Godiska R, Shanker S, Yu F, Farmerie WG, Ashizawa T. SMRT sequencing of long tandem nucleotide repeats in SCA10 reveals unique insight of repeat expansion structure. PLoS One. 2015;10(8): e0135906.

23. Ardui S, Race V, Zablotskaya A, Hestand MS, Van Esch H, Devriendt K, Matthijs G, Vermeesch JR. Detecting AGG interruptions in male and female FMR1 Premutation carriers by single-molecule sequencing. Hum Mutat. 2017;38:324-31.

24. UCSC Sequence and Annotation Downloads, Feb. 2009 Assembly of the human genome. http://hgdownload.soe.ucsc.edu/goldenPath/hg19/ chromosomes/chrX.fa.gz. Accessed 10 Sept 2013.

25. Rice $\mathrm{P}$, Longden I, Bleasby A. EMBOSS: the European molecular biology open software suite. Trends Genet. 2000;16(6):276-7.

26. UCSC Sequence and Annotation Downloads, Feb. 2009 Assembly of the human genome. http://hgdownload.soe.ucsc.edu/goldenPath/hg19/ database/microsat.txt.gz. Accessed 10 Sept 2013.

27. Short Tandem Repeat DNA Internet DataBase. http://www.cstl.nist.gov/ biotech/strbase/. Accessed 10 Sept 2013.

28. Orr HT, Zoghbi HY. Trinucleotide repeat disorders. Annu Rev Neurosci. 2007; 30:575-621.

29. UCSC Sequence and Annotation Downloads, Feb. 2009 Assembly of the human genome. http://hgdownload.soe.ucsc.edu/goldenPath/hg19/ database/simpleRepeat.txt.gz. Accessed 10 Sept 2013.

30. Duitama J, Zablotskaya A, Gemayel R, Jansen A, Belet S, Vermeesch JR, Verstrepen KJ, Froyen G. Large-scale analysis of tandem repeat variability in the human genome. Nucleic Acids Res. 2014;42(9):5728-41. 
31. Greenwood Genetic Center. https://www.ggc.org/xlid-genetic-research. Accessed 20 Apr 2014.

32. Langmead B, Trapnell C, Pop M, Salzberg SL. Ultrafast and memory-efficient alignment of short DNA sequences to the human genome. Genome Biol. 2009;10:R25

33. Claes $\mathrm{S}$, Volcke $\mathrm{P}$, Devriendt $\mathrm{K}$, Holvoet M, Raeymaekers $\mathrm{P}$, Cassiman JJ, Fryns JP. Regional localization of a gene for nonspecific XLMR to Xp11.3-p11.23 (MRX51) and tentative localization of an MRX gene to Xq23-q26.1. Am J Med Genet. 1999, 85:283-7.

34. Li H, Durbin R. Fast and accurate long-read alignment with burrowswheeler transform. Bioinformatics. 2010;26(5):589-95.

35. Picard. http://broadinstitute.github.io/picard. Accessed 14 Aug 2014.

36. Quinlan AR, Hall IM. BEDTools: a flexible suite of utilities for comparing genomic features. Bioinformatics. 2010;26(6):841-2.

37. Anvar SY, van der Gaag KJ, van der Heijden JW, Veltrop MH, Vossen RH, de Leeuw RH, Breukel C, Buermans HP, Verbeek JS, de Knijff P, et al. TSSV: a tool for characterization of complex allelic variants in pure and mixed genomes. Bioinformatics. 2014;30(12):1651-9.

38. Rozen S, Skaletsky H. Primer3 on the WWW for general users and for biologist programmers. In: Krawetz S, Misener S, editors. Bioinformatics methods and protocols: methods in molecular biology. Totowa, N.J: Humana Press; 2000. p. 365-86.

39. Trapnell C, Pachter L, Salzberg SL. TopHat: discovering splice junctions with RNA-Seq. Bioinformatics. 2009;25(9):1105-11.

40. Li H, Handsaker B, Wysoker A, Fennell T, Ruan J, Homer N, Marth G, Abecasis G, Durbin R. 1000 genome project data processing subgroup. The Sequence alignment/map (SAM) format and SAMtools. Bioinformatics. 2009;25(16): 2078-9.

41. Trapnell C, Williams BA, Pertea G, Mortazavi A, Kwan G, van Baren MJ, Salzberg SL, Wold BJ, Pachter L. Transcript assembly and quantification by RNA-Seq reveals unannotated transcripts and isoform switching during cell differentiation. Nat Biotechnol. 2010;28:511-5.

42. Delphin N, Hanein S, Taie LF, Zanlonghi X, Bonneau D, Moisan J-P, Boyle C, Nitschke P, Pruvost S, Bonnefont J-P, et al. Intellectual disability associated with retinal dystrophy in the Xp11.3 deletion syndrome: ZNF674 on trial. Guilty or innocent? Eur J Hum Genet. 2012;20(3):352-6.

43. Rio M, Malan V, Boissel S, Toutain A, Royer G, Gobin S, Morichon-Delvallez N, Turleau C, Bonnefont J-P, et al. familial interstitial Xq27.3q28 duplication encompassing the FMR1 gene but not the MECP2 gene causes a new syndromic mental retardation condition. Eur J Hum Genet. 2010;18(3):285-90.

44. Vengoechea J, Parikh AS, Zhang S, Tassone F. De novo microduplication of the FMR1 gene in a patient with developmental delay, epilepsy and hyperactivity. Eur J Hum Genet. 2012;20(11):1197-200.

45. Nagamani SCS, Erez A, Probst FJ, Bader P, Evans P, Baker LA, Fang P, Bertin $T$, Hixson P, Stankiewicz $P$, et al. Small genomic rearrangements involving FMR1 support the importance of its gene dosage for normal neurocognitive function. Neurogenetics. 2012;13:333.

46. Hickey SE, Walters-Sen L, Mosher TM, Pfau RB, Pyatt R, Snyder PJ, Sotos JF, Prior TW. Duplication of the Xq27.3-q28 region, including the FMR1 gene, in an X-linked hypogonadism, gynecomastia, intellectual disability, short stature, and obesity syndrome. Am J Med Genet Part A. 2013;161A:2294-9.

47. Guilmatre A, Highnam G, Borel C, Mittelman D, Sharp AJ. Rapid multiplexed genotyping of simple tandem repeats using capture and high-throughput sequencing. Hum Mutat. 2013;34:1304-11.

48. Mclver LJ, McCormick JF, Martin A, Fondon JW III, Garner HR. Populationscale analysis of human microsatellites reveals novel sources of exonic variation. Gene. 2013;516:328-34.

49. Willems T, Gymrek M, Highnam G. The 1000 genomes project consortium, Mittelman D, Erlich Y. the landscape of human STR variation. Genome Res. 2014;24(11):1894-904.

50. Yoon K, Lee S, Han TS, Moon SY, Yun SM, Kong SH, Jho S, Choe J, Yu J, Lee $\mathrm{HJ}$, et al. Comprehensive genome- and transcriptome-wide analyses of mutations associated with microsatellite instability in Korean gastric cancers. Genome Res. 2013;23:1109-17.

51. Fondon JW III, Martin A, Richards S, Gibbs RA, Mittelman D. Analysis of microsatellite variation in Drosophila melanogaster with population-scale genome sequencing. PLoS One. 2012;7:e33036.

52. Malausa T, Gilles A, Meglecz E, Blanquart H, Duthoy S, Costedoat C, Dubut V, Pech N, Castagnone-Sereno P, Delye C, et al. High-throughput microsatellite isolation through 454 GS-FLX titanium pyrosequencing of enriched DNA libraries. Mol Ecol Resour. 2011;11:638-44.
53. Altemose N, Miga KH, Maggioni M, Willard HF. Genomic characterization of large heterochromatic gaps in the human genome assembly. PLoS Comput Biol. 2014;10(5):e1003628.

54. Chaisson MJP, Huddleston J, Dennis MY, Sudmant PH, Malig M, Hormozdiari F, Antonacci F, Surti U, Sandstrom R, Boitano M, et al. Resolving the complexity of the human genome using single-molecule sequencing. Nature. 2015;517(7536): 608-11.

55. Miga KH. Completing the human genome: the progress and challenge of satellite DNA assembly. Chromosom Res. 2015;23:421.

56. Granok H, Leibovitch BA, Shaffer CD, Elgin SCR. Ga-ga over GAGA factor. Curr Biol. 1995;5(3):238-41.

57. Lehmann M. Anything else but GAGA: a nonhistone protein complex reshapes chromatin structure. Trends Genet. 2004;20(1):15-22.

58. Tsai S-Y, Chang Y-L, Swamy KBS, Chiang R-L, Huang D-H. GAGA factor, a positive regulator of global gene expression, modulates transcriptional pausing and organization of upstream nucleosomes. Epigenetics Chromatin. 2016;9:32.

59. Emamalizadeh B, Movafagh A, Darvish H, Kazeminasab S, Andarva M, Namdar-Aligoodarzi P. Ohadi M. The human RIT2 core promoter short tandem repeat predominant allele is species-specific in length: a selective advantage for human evolution? Mol Gen Genomics. 2017;438:1-7.

60. Valipour E, Kowsari A, Bayat H, Banan M, Kazeminasab S, Mohammadparast $\mathrm{S}$, Ohadi M. Polymorphic core promoter GA-repeats alter gene expression of the early embryonic developmental genes. Gene. 2013;531(2):175-9.

61. Berger N, Dubreucq B. Evolution goes GAGA: GAGA binding proteins across kingdoms. Biochim Biophys Acta Gene Regul Mech. 2012;1819(8):863-8.

62. Nakayama T, Nishioka K, Dong Y-X, Shimojima T, Hirose S. Drosophila GAGA Factor directs histone $\mathrm{H} 3.3$ replacement that prevents the heterochromatin spreading. Genes Dev 2007;21(5):552-561.

63. Kapsimali M, Kloosterman WP, de Bruijn E, Rosa F, Plasterk RH, Wilson SW. MicroRNAs show a wide diversity of expression profiles in the developing and mature central nervous system. Genome Biol. 2007;8(8):R173.

64. Medina R, Zaidi SK, Liu C-G, Stein JL, van Wijnen AJ, Croce CM, Stein GS. MicroRNAs 221 and 222 bypass quiescence and compromise cell survival. Cancer Res. 2008;68(8):2773-80.

65. Podolska A, Kaczkowski B, Kamp Busk P, Sokilde R, Litman T, Fredholm M, Cirera S. MicroRNA expression profiling of the porcine developing brain. PLoS One. 2011;6(1):e14494.

66. Decembrini S, Bressan D, Vignali R, Pitto L, Mariotti S, Rainaldi G, Wang X, Evangelista M, Barsacchi G, Cremisi F. MicroRNAs couple cell fate and developmental timing in retina. Proc Natl Acad Sci U S A. 2009;106(50): 21179-84.

67. Chen W, Jensen LR, Gecz J, Fryns J-P, Moraine C, de Brouwer A, Chelly J, Moser B, Ropers HH, Kuss AW. Mutation screening of brain-expressed Xchromosomal miRNA genes in 464 patients with nonsyndromic X-linked mental retardation. Eur J Hum Genet. 2007:15:375-8.

68. Kan AA, van Erp S, Derijck AAHA, de Wit M, Hessel EVS, O'Duibhir E, de Jager W, Van Rijen PC, Gosselaar PH, de Graan PNE, et al. Genome-wide microRNA profiling of human temporal lobe epilepsy identifies modulators of the immune response. Cell Mol Life Sci. 2012;69(18):3127-45.

69. Zhoua L-T, Yea S-H, Yanga H-X, Zhoua Y-T, Zhaoa Q-H, Suna W-W, Gaoa M$\mathrm{M}$, Yia Y-H, Longa $Y$-S. A novel role of fragile $X$ mental retardation protein in pre-mRNA alternative splicing through RNA-binding protein. Neuroscience. 2017;349:64-75.

70. Raj B, Blencowe BJ. Alternative splicing in the mammalian nervous system: recent insights into mechanisms and functional roles. Neuron. 2015;87(1):14-27.

71. Peier AM, Mcllwain KL, Kenneson A, Warren ST, Paylor R, Nelson DL. (over)correction of FMR1 deficiency with YAC transgenics: behavioral and physical features. Hum Mol Genet. 2000;9(8):1145-59.

72. Al-Chalabi A, Miller CCJ. Neurofilaments and neurological disease. BioEssays. 2003:25:346-55.

73. Collard J-F, Cote F, Julien J-P. Defective axonal transport in a transgenic mouse model of amyotrophic lateral sclerosis. Nature. 1995;375:61-4.

74. Qin L, Liu X, Liu S, Liu Y, Yang Y, Yang H, Chen Y, Chen L. Differentially expressed proteins underlying childhood cortical dysplasia with epilepsy identified by iTRAQ proteomic profiling. PLoS One. 2017;12(2):e0172214.

75. Lv Q, Lai L, Yuan L, Song Y, Sui T, Li Z. Tandem repeat knockout utilizing the CRISPR/Cas9 system in human cells. Gene. 2016;582(2):122-7.

76. Van Agtmaal EL, André LM, Willemse M, et al. CRISPR/Cas9-induced (CTG.CAG)n repeat instability in the myotonic dystrophy type 1 locus: implications for therapeutic genome editing. Mol Ther. 2017;25(1):24-43. 